\title{
LA EDUCACIÓN AMBIENTAL: UNA TAREA INCONCLUSA DESDE LOS PROYECTOS AMBIENTALES ESCOLARES
}

\section{ENVIRONMENTAL EDUCATION: AN UNFINISHED TASK IN SCHOOL ENVIRONMENTAL PROJECTS}

\author{
Nabi Del Socorro Pérez-Vásquez 1 \\ Edith De Jesús Cadavid-Velásquez ${ }^{2}$ \\ Elvira Patricia Flórez-Nisperuza ${ }^{3}$ \\ Universidad de Córdoba
}

\section{RESUMEN}

La Educación Ambiental (EA) en Colombia se institucionalizó de manera efectiva a partir de la promulgación del Decreto 1743 de 1994,

$\overline{1}$ Doctora en Educación y Cultura Ambiental. Magister en Ciencias Ambientales. Especialista en Docencia Universitaria. Licenciada en Biología y Química. Docente Universidad de Córdoba. E mail: ndperezvasquez@correo.

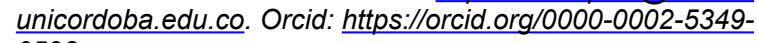
$\underline{0598}$

$2 \quad$ Magister en Ciencias Ambientales. Especialista en Etnobiología. Licenciada en Biología y Química. Docente Universidad de Córdoba. E mail: edithcadavid@correo.unicordoba.edu.co. Orcid: https://orcid.org/0000-0003-0115$\underline{8857}$

3 Doctora en Ciencias de la Educación. Magister en Educación y Desarrollo Humano. Especialista en Pedagogía y Didáctica. Licenciada en Química y Biología. Docente Universidad de Córdoba. Email: epatriciaflorez@correo.unicordoba.edu.co. Orcid: https://orcid.org/0000-0003-4621-8382 mediante los Proyectos Ambientales Escolares (PRAEs) como una de las estrategias de formación ambiental, así, su formulación y ejecución debe fundamentarse como un componente curricular que tienda al desarrollo de valores, desde la interdisciplinariedad, la participación, la formación para la democracia, la gestión y la resolución de problemas del contexto regional. En la investigación se caracterizó el estado actual de los PRAEs de las Instituciones Educativas (IE) oficiales urbanas y rurales de educación básica y media del municipio de Montería, en relación con los requerimientos normativos en su construcción y su dinamización. Mediante una revisión documental, utilizando 
para el análisis una matriz porcentual de Excel y apoyado en entrevistas a grupos focales de docentes las cuales se analizaron desde el paradigma de la hermenéutica -interpretativa. Los resultados evidencian que, en la mayoría de las IE, los proyectos se ejecutan de manera aislada a la gestión académica, dejando de lado principios didácticos de transversalidad, interdisciplinariedad e integralidad del currículo y generalmente liderados por los profesores de Ciencias Naturales. Se concluye que estos proyectos no han permitido consolidar una educación ambiental integral que tengan en cuenta los aspectos naturales y socioculturales y el mejoramiento de la calidad de las comunidades educativas.

PALABRAS CLAVE: Educación ambiental, instituciones educativas, cultura ambiental, normatividad.

\section{ABSTRACT.}

Environmental Education (EA) in Colombia was effectively institutionalized as of the promulgation of Decree 1743 of 1994, through School Environmental Projects (PRAEs) as one of the environmental training strategies, thus, its formulation and execution must be based on a curricular component that tends to develop values, from interdisciplinarity, participation, training for democracy, management, and resolution of problems in the regional context. The research characterized the current state of the PRAEs of the official urban and rural Educational Institutions (IE) of basic and secondary education of the municipality of Montería, in relation to the regulatory requirements for their construction and dynamization. Through a documentary review, using an Excel percentage matrix for analysis and supported by interviews with focus groups of teachers, which were analyzed from the hermeneutic-interpretive paradigm. The results show that, in most Els, projects are executed in isolation from academic management, leaving aside didactic principles of transversality, interdisciplinarity and comprehensiveness of the curriculum and generally led by Natural Sciences teachers. It is concluded that these projects have not made it possible to consolidate a comprehensive environmental education that takes into account natural and socio-cultural aspects and the improvement of the quality of educational communities.

KEYWORDS: Environmental education, educational institutions, environmental culture, regulations.

\section{INTRODUCCIÓN}

La Educación Ambiental, demanda contribuir a la formación de un nuevo ciudadano, que lleve a una relación armónica con el entorno y con los demás componentes, que responda a la actual crisis ambiental asociada a la modernidad capitalista, crisis de sentido, que fragmentó el mundo "gobernado por la razón y la economía que crece sin límites se alimenta de naturaleza, de materias y energía, de biodiversidad, de formas de vida y de modos de existencia" (Leff, 2019) la cual ha conducido a problemáticas socioambientales que amenazan la supervivencia en y del planeta. Por ello, sus contenidos y estrategias pedagógicas se fundamentarán en el desarrollo conceptual, teórico y práctico de un saber ambiental que se nutran de la acción concreta, tanto en los procesos de formación y de organización de los actores sociales del desarrollo sustentable, como en la construcción y gestión de procesos democráticos" (Leff, 2008, p.113). La EA posibilita la formación humana capaz de comprender la complejidad del ambiente, en la interacción de sus componentes naturales y socioculturales, a la vez que le permite "ser crítico, emitir juicios de valor y adoptar normas de comportamiento en actividades integradas en un proceso sistemático y permanente" (Pasek, 2004, p.36). 
Es de anotar que, en Colombia el PRAE, se constituye en una de las estrategias de educación ambiental institucional. En el Decreto 1743 se establece que "todos los establecimientos de educación formal del país, tanto oficiales como privados, en sus distintos niveles de preescolar, básica y media, incluirán los PRAEs dentro de sus proyectos educativos institucionales, partiendo de diagnósticos ambientales, locales, regionales y/o nacionales, con miras a coadyuvar a la resolución de problemas ambientales específicos". La instrucción normativa obedece a la necesidad de conceptuar, contextualizar y operacionalizar la educación ambiental con la formación integral tanto de la comunidad educativa y su entorno, como principios rectores que deben estar presentes en todos los componentes del currículo.

Reafirmando lo anterior, la Política Nacional de Educación Ambiental (PNEA), concibe el PRAE como una de las estrategias pedagógicas clave para desarrollar la formación ambiental y comprender mejor el territorio y la sociedad colombiana, así como plantear conjuntamente soluciones a los problemas ambientales que los aqueja. Asimismo, se conciben cuatro etapas fundamentales en el desarrollo de la educación ambiental: la exploración, donde se identifican los actores comprometidos; la profundización, en la que se conceptualiza la educación ambiental; la proyección, que busca llegar a acuerdos efectivos de educación ambiental a través de la concertación y las estrategias, apoyadas en los lineamientos y criterios sobre los cuales se aborda la educación ambiental. Al respecto, Flórez-Restrepo (2012) exaltó la importancia del desarrollo de la Educación Ambiental en los entornos educativos formales, no formales e informales, en los que estrategias como los PRAEs deben movilizar a las comunidades en pro de la comprensión-acción de una realidad ambiental esta apuesta de la Educación Ambiental trasciende las esferas propiamente escolares y se convierte en el escenario comunitario para compartir, interactuar.

La PNEA, promulga que la EA debe comprometerse con la formación de ciudadanos éticos, responsables, capaces de comprender la realidad social y natural, hábiles para intervenir de manera participativa, consciente y crítica en el logro de unas relaciones sociedad-naturaleza caracterizadas por la sostenibilidad. Sin embargo, los PRAEs en algunas instituciones, se han diseñado y ejecutado de manera estandarizada, sin proyección comunitaria, ni reflexión epistemológica, política, ética y social. En este sentido, Mora Ortiz (2015) destacó que, en los PRAEs, se muestra poca o nula participación comunitaria, siendo también limitado en el componente de gestión ambiental local.

En el desarrollo y dinamización de los proyectos escolares se puede observar en la práctica educativa quedan desprovisto del carácter sistémico e interdisciplinario se desarrollan en la base de un activismo ecológico mediático, campañas de aseo, manejo o transformación de residuos, repetición de frases y conductas, que en muchos casos resultan insuficientes, toda vez que no generan cambios profundos en la forma de actuar, sentir y pensar de la comunidad educativa (Pérez- Vásquez, 2020), por ello es necesario propiciar escenarios para el diálogo, la discusión y la reflexión colectiva, con miras a generar una construcción social del conocimiento del saber ambiental, ubicando éste desde la diversidad y la complementariedad (Flórez Restrepo, 2012 p. 82). Por su parte Burgos-Ayala (2017) al investigar el estado de los PRAEs en Boyacá, acentuó que éstos no abordan los problemas del territorio de la zona de vida de las IE; sino que priorizan temas de concientización, pero en las acciones persiste el manejo de residuos sólidos y el reciclaje, desconociendo otro tipo de conflictos de mayor impacto. 
En el contexto de la región Caribe, Bustamante, Cruz y Vergara (2017), realizaron la caracterización de los PRAEs y la cultura ambiental de los estudiantes en Sincelejo, con el propósito de ofrecer alternativas de mejoramiento. En el manifiestan que los docentes reconocen como limitantes a la falta de compromiso, los escases de recursos económicos, espacio físico inadecuado, falta de coordinación y el desarrollo de reuniones poco estructuradas y los estudiantes atribuyen las limitaciones al espacio físico, la falta de recurso humano, el tiempo atribuido al PRAE, Falta de compromiso, falta de asesoría y escasa coordinación. Mientras que los directivos observan menores.

En el ámbito local, Villadiego, Cardona, Ortiz, Coneo y Ramos en el 2011 realizaron un análisis de la implementación de los PRAEs en el departamento de Córdoba y determinaron que la principal línea temática es el manejo de residuos sólidos, la mayoría conoce la Política Nacional de Educación Ambiental (PNEA, 2002) y en poca medida las IE usan el PRAE como principal estrategia de educación ambiental. Desde la situación comentada, parte la necesidad de evaluar en el año 2019 los procesos educativoambientales asociados a los PRAEs de las IE de la ciudad de Montería-Córdoba-Colombia en relación con lo establecido normativamente, analizando su naturaleza contextual, conceptual y estructural, en concordancia con las dinámicas curriculares y el desarrollo efectivo de una educación ambiental significativa en la escuela, que genere nuevos acercamientos a sus distintos integrantes y propuestas de articulación y apropiación en estos campos. El aporte reside además en que brinda elementos en la unificación estructural de los PRAEs a partir de un instructivo para su construcción, apoyados en el diagnóstico, la contextualización de la problemática, la proyección social comunitaria y la articulación de estos con el PEI, el POT y los planes de desarrollo municipal, en aras de lograr una EA desde lo local con proyección regional y nacional.

Este estudio se constituye un nuevo escenario del quehacer investigativo de la Universidad de Córdoba directamente asociado al grupo de investigación GICNEA, categoría $B$ de Colciencias, proyectado para unir lazos diagnósticos y proyectivos en relación con el estado actual de los PRAEs en respuesta a lo establecido normativamente y acercamientos cualitativos con docentes de las instituciones educativas y plantar a partir de ello, acciones conjuntas con la seguridad puesta en desarrollar procesos de transformación hacia el mejoramiento de la dinámica escolar desde una permanente interacción entre los miembros de la comunidad educativa.

\section{METODOLOGÍA.}

La investigación se desarrolló desde la hermenéutica- interpretativa, de acuerdo con su sentido más tradicional, se relaciona con el arte de interpretar, es ciencia porque contiene un conjunto de principios y reflexiones teóricas en torno a la interpretación y es técnica, porque regula la forma en que se ha de interpretar algo (Monzón, 2011).

El estudio, se desarrolla en tres fases, para efectos del artículo se destacan dos, la fase exploratoria a través de la técnica de revisión documental en la que se caracterizaron los 59 PRAEs de las IE oficiales urbanas y rurales de educación básica y media del municipio de Montería, interpretados en correspondencia con una matriz de evaluación propuesta por el Ministerio de Educación Nacional (MEN, 2011) en relación con el cumplimiento de los requerimientos normativos apoyados en una matriz de Excel que se muestra de manera porcentuales y la fase interpretativa desde el análisis de entrevistas a grupo focales de docentes, los cuales se codificados para responder a consideraciones éticas de 
confidencialidad como docentes de Ciencias Naturales (DCN), de Ciencias Sociales (DCS), de Preescolar (DP), de Primaria (DPR), de Informática (DI) de Artística (DA), de Lengua Castellana (DLC).

\section{RESULTADOS Y DISCUSIÓN.}

De acuerdo a lo referenciado teóricamente a lo largo del artículo, el PRAE es una herramienta importante en la apertura de espacios de desarrollo de la intervención - investigación, si se tiene en cuenta que el objeto del mismo, es la comprensión de las realidades del territorio, sus problemáticas y/o potencialidades ambientales, a través de la construcción de conocimientos significativos que redunden en beneficio de la cualificación de las actitudes y de los valores, en el manejo adecuado del ambiente, no solo para el desarrollo de acciones locales, sino hacia la generación de conciencia ambiental que a futuro, aseguren actitudes de conservación de los ecosistemas en cualquiera de los ámbitos de su vida.

Al analizar el estado actual de los PRAEs de las instituciones públicas del municipio de Montería, se evidenció que el $98.2 \%$ (58) de los proyectos analizados se encuentran en ejecución; sin embargo, solo $59.3 \%$ (35) muestra evidencias prácticas de dinamización, es decir, se pueden considerar proyectos de papel, bien concebidos pero convertidos en letra muerta lo que implica que no se desarrolla una formación ambiental trascendental en la vida de los estudiantes en y fuera del aula, por lo que consideramos que la tarea de la Educación Ambiental queda inconclusa.

Del mismo modo, la gran mayoría es liderado por el Área de Ciencias Naturales, aunque el $57.6 \%$ (34) reconoce que se trabaja en conjunto, en correspondencia con ello los docentes manifiestan "el PRAE es liderado por los profesores de Ciencias naturales" (DCS, DLC, DI, DCN, DP, DPR), con poca participación de otras áreas de formación, solo dos entrevistados mencionaron la participación efectiva de varias áreas académicas en la implementación, de hecho reconocen que "no ha sido fácil, se ha logrado luego de varios años, al principio hubo bastante resistencia" (DCN).

Al contrastar esta situación con lo planteado en algunas investigaciones la situación es semejante, en tanto, señalan que la participación docente, además de ser limitada, se restringe en su mayoría a áreas relacionadas con las ciencias naturales (Burgos, 2017, p 53), lo que puede estar asociado con el desconocimiento de las maneras de participación curricular desde la interdisciplinariedad y transversalidad, además, de acuerdo a algunos docentes que participaron en las entrevistas "es una carga más, porque la planeación de las actividades debe hacerse en horario contraria o contra jornada" (DCN), "es difícil que los padres permitan a los estudiantes estar en todas las actividades por que muchas de hacen en horas extra clase" (DLC), lo que al final se constituye en una de las principales barrera para la dinamización e implementación de los proyectos.

De los proyectos analizados un $40.7 \%$ (24) abordan la gestión de residuos, es decir su ejecución se hace solo al interior de la IE, sin hacer una adecuada lectura de las necesidades reales de la comunidad. Lo cual fue confirmado por los docentes entrevistados, "el proyecto se asocia a la gestión de residuos y la sensibilización a la comunidad de la importancia de la educación ambiental y aspectos sociales" (DCS); "el proyecto se ha fundamentado en la gestión de residuos" (DCN); "en la institución del proyecto se ha abordado a partir la gestión de residuos" (DI).

Un 39\% (23) aborda temas relacionados con la cultura ambiental, sin embargo las pocas evidencias que se incluyen en el documento permite ver que se asociada a la misma temática de gestionar residuos e incluso los 
transforman o los venden y logran ganar dinero que se invierte según los entrevistados en el embellecimiento del colegio; aspecto que había sido reportado por Villadiego et al. (2011, p12), quienes reconocieron en su estudio que más de la mitad de los PRAEs estudiados apunta al manejo de residuos, lo que evidencia que la estrategia solo se desarrolla al interior de las instituciones y no se articula con la comunidad, siendo de la misma manera en la actualidad, al explorar las causas, los entrevistados reconocen que se debe al facilismo y al hecho de que el tiempo escolar es insuficiente para trabajarlo en la jornada académica correspondiente.

Otros de los temas abordados en menor porcentaje son la dimensión ambiental en un $6.8 \%$ (4), el ornato y aseo en el $5.1 \%$ (3) de los casos que se relaciona con campañas desarrolladas especialmente cuando hay conmemoraciones especiales en el colegio, lo cual no permite la profundización del saber y la significancia de este. De otro lado, un $3.4 \%$ (2) de los proyectos tienen como tema prioritario la conservación de recursos naturales, PRAEs fundamentado en la protección, conservación y aprovechamiento de los recursos naturales del entorno de manera responsable y sostenible y la conservación y sostenibilidad de la fauna silvestre, algo para resaltar es que son instituciones de carácter rural, lo que probablemente ha llevado a tomar como foco central del proyecto estas actividades.

Finalmente, el resto de los proyectos, que corresponde a menos del $2 \%$ (1), dedican sus ejecuciones a la sustentabilidad, la seguridad alimentaria, a la conformación de un grupo ecológico, como se sintetiza en Figura 1 y 2. Temas que se consideran de relevancia, lo cual podría dar significancia al PRAEs, en tanto este debe propiciar en el estudiantado, el desarrollo de competencias básicas y ciudadanas, para que tomen decisiones de manera ética y responsable frente al manejo y cuidado del ambiente (Andrade y Andrade, 2017) y a la resolución de los problemas del contexto sin embargo, los resultados muestran un bajo porcentaje, de ahí que es necesario fomentar el desarrollo de proyectos que hagan una debida lectura de contexto para que exista 
Figura 1. Estructuración general de los PRAEs

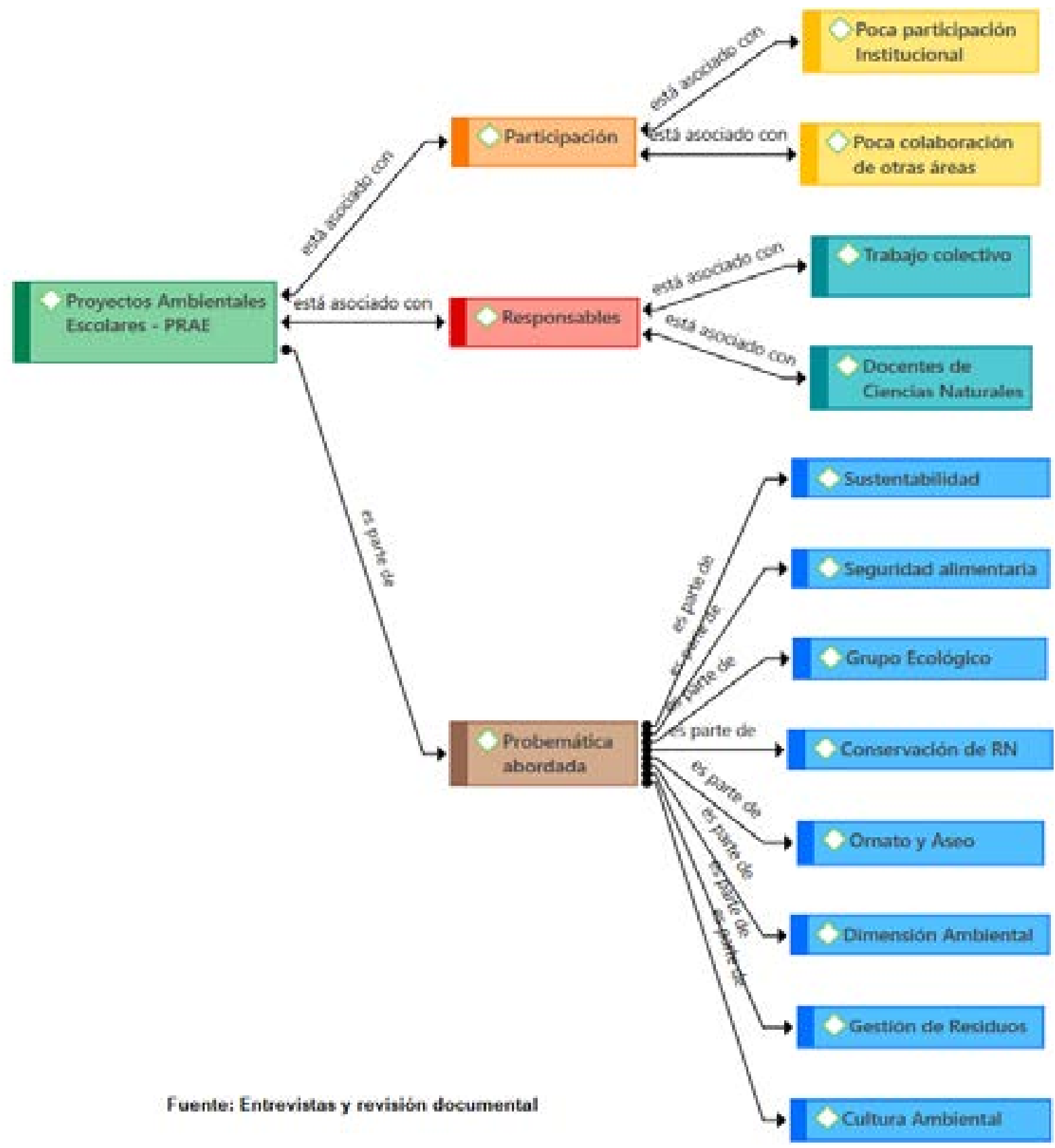


Figura 2. Porcentaje de Problemáticas abordadas en los PRAEs de las IE de Montería

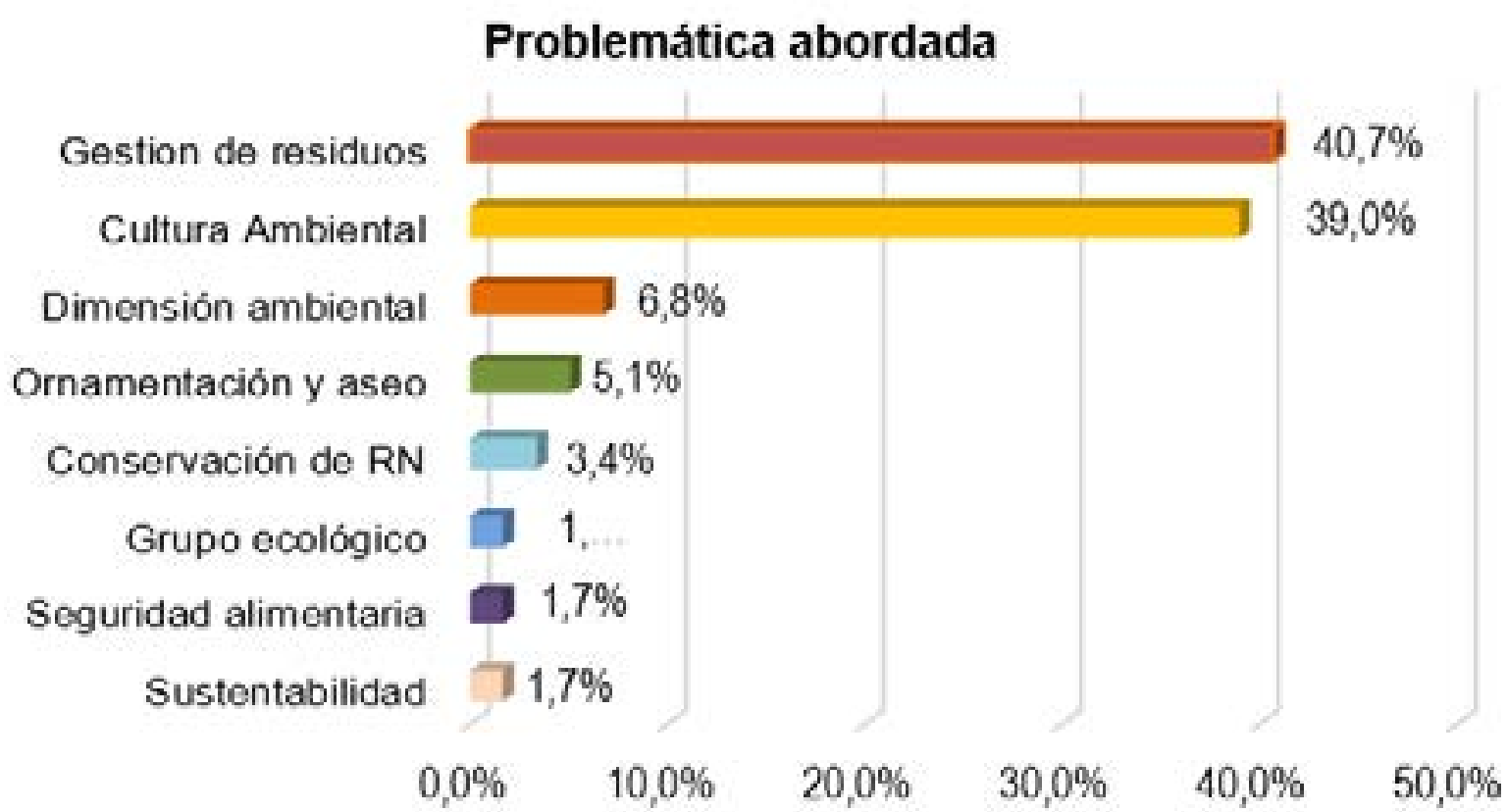

Fuente: Procesamiento de los datos obtenidos en la investigación

Complementando lo anterior, los docentes entrevistados reconocen que la dinamización del PRAE "se queda en acciones asiladas, activismo ambiental" (DCS), relacionado a "conmemoración de fechas ambiental, no profundizan en la concienciación ambiental" (DP), se hacía "siembra de árboles una o dos veces al año" (DCN); "se hacen varias actividades, celebración de fechas ambientales, campañas de limpieza, reinado, trabajos manuales, sin embargo falta la sistematización de las actividades donde se destaquen procesos de evaluación, solo se recogen las evidencias fotográficas" (DA). Tal como lo destaca Eschenhagen (2003, p.12) abordan soluciones a los problemas desde acciones en muchos casos instrumentales y técnicas las IEs suscitan un activismo inmediatista ciego, sin reflexiones profundas y críticas del cómo, el por qué y el para qué la Educación Ambiental este actuar, conlleva a posiciones instrumentalistas y tecnologistas. Lo que claramente se constata en los documentos analizados no existe un seguimiento evaluativo que permita fortalecer los procesos educativo-ambientales y que propendan por un cambio fundamental de creencias, actitudes y estilos de vida de los estudiantes.

Estos proyectos en su gran mayoría abordan temas institucionales, es decir, dejan de lado lo estatuido por norma en relación con la proyección social, en tanto, el $82 \%$ (48) no reconocen, ni describen actividades sociales y económicas de la comunidad, tal como se destaca en la PNEA (2002, p. 12) al reconocer que la concentración de los trabajos educativo ambientales se asocian a aspectos puramente ecológicos, dejando de lado los aspectos culturales y sociales que hacen parte integral de la problemática ambiental. Asimismo, ninguno de los PRAEs analizados muestra articulación con otros procesos locales de educación ambiental como los Proyectos Ciudadanos de Educación Ambiental como está estatuido en el marco normativo y en la política. 
Además, el análisis de los PRAEs caracterizados no muestra la relación entre éstos con los instrumentos de ordenamiento y desarrollo territorial (POT) y mucho menos con Plan de Desarrollo Municipal evidenciándose una descontextualización entre las acciones de la EA, debido a que éstas no parten de los diagnósticos o perfiles regionales y locales, ni se relacionan con los planes regionales ambientales, para éste mismo aspecto, falta proyección de la visión sistémica del ambiente a los análisis de los problemas ambientales (PNEA, 2002, p.11).

En los documentos oficiales se destacan que un $93.2 \%$ (55) describe el problema ambiental que abordara a través del PRAE, acentuando sus causas, quedando en una descripción poco detallada y sin análisis profundo, siendo notorio que sólo 42,4\% (25) muestra las consecuencias del problema. Atendiendo al aspecto conceptual de los documentos oficiales de los PRAEs, emergen elementos a destacar; por ejemplo, no existe una estructura única en la construcción de este, dado que no se ciñen a lo que se establece por norma, de ahí que se encuentren documentos densamente elaborados y algunos con una pobreza conceptual y metodológica.

Así, se destaca que solo el $22,2 \%$ (13) conceptualizó teóricamente el tema central del PRAE desde referentes bibliográficos, en el concepto de ambiente solo el $28,8 \%$ (17) y el de la educación ambiental el 30,5\% (18), sustentabilidad el $6,8 \%$ (4) y otras categorías que se destacan normativamente como gestión, desarrollo y proyección comunitaria no son tenidos en cuenta en los documentos de los proyectos (Figura 3).

Figura 3. Incorporación de referentes teóricos en las categorías conceptuales de los PRAEs

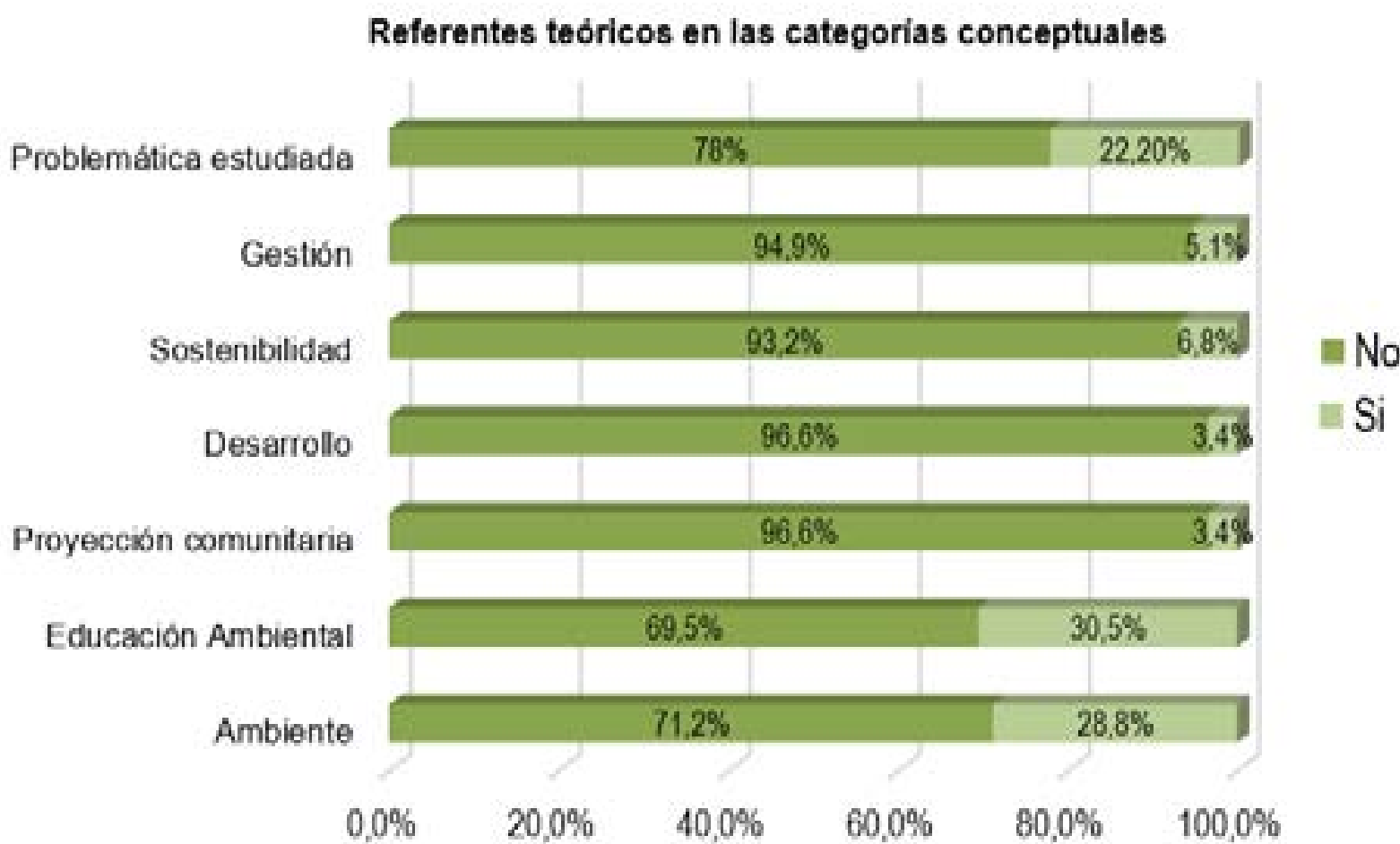

Fuente: Procesamiento de datos obtenidos de la investigación 
En este sentido, se evidencia que en su gran mayoría no utilizan fundamentos teóricos amplios en cada una de las categorías conceptuales de los temas abordado en los proyectos, se quedan en meras conceptualizaciones básicas. Así lo destacó Burgos (2013) al señalar que los PRAEs parten de una pobre concepción de lo qué son, para qué son, y/o a quién corresponde su ejecución en la IE, se desconoce su finalidad y sus objetivos formativos, asociados a la generación de la conciencia ambiental de los individuos en el contexto, mediante el reconocimiento y aporte a realidades ambientales locales, además, docentes y estudiantes lo toman como algo pasajero, impuesto normativamente y en el mejor de los casos, como algo acciones aisladas que se constituyen en activismo ambiental.

En relación con elementos estructurales de los 59 PRAEs revisados, se destaca que solo el $6,77 \%$ (11) detalla en sus propósitos generales y específicos en el marco del PEI y el desarrollo local y regional, resultados que confirman las brechas y las lejanías de los planteamientos institucionales. Según González Pachón (2014), el PRAE debe reconocer y plasmar a través de sus actividades la búsqueda de la coherencia entre lo que se planea y se ejecuta; fortalecer los procesos de argumentación acerca de los quehaceres. En ocasiones el PEI hace más realce en formación en valores y no muestra claramente la inclusión de estos.

Un segundo elemento estructural fruto de la revisión documental, es lo relacionado con la identificación de los enfoques pedagógicos y didácticos de los PRAEs desde los propósitos de la educación ambiental. Solo el 11,8\% (20) $y$ el $18,64 \%$ (11) de los documentos oficiales de las instituciones educativas identifica estos parámetros respectivamente. Vale anotar que las confirmaciones reveladas en los documentos si bien precisan en los enfoques de aula a seguir, están desprovistos de una sólida fundamentación epistemológica, conceptual y teórica; y por su parte, revelan un conjunto de acciones encaminadas a organizar ejercicios de ambientalización concordantes con las problemáticas ambientales detectadas y que ratifican que a pesar de que existen lineamientos desde 1994 (Decreto 1743) y la PNEA, hace falta claridad en la ubicación de los procesos pedagógico-didácticos, como factores clave en el campo axiológico en la educación ambiental y en la transformación de la dinámica educativa del país (Torres-Carrasco, 2002).

Un tercer elemento estructural, atiende la propuesta interdisciplinaria de los PRAEs revisados, derivando importantes señalamientos, dado que solo el $13 \%$ (8) del total, precisa en una intención de integrar las áreas al debate de la educación ambiental. Esta cifra sorprende aún más, toda vez que se asumen los PRAEs de las instituciones Educativas registradas, la interdisciplinaridad como sinónimo de participar integradamente en actividades ambientales, operativizando la educación ambiental y olvidando los postulados conceptuales y metodológicos propios de este imperativo curricular, entendida como "lo que se da entre varios" en un campo de formación específica, que implica la interacción de disciplinas a partir del diálogo de saberes y competencias. Estos procesos exigen una planeación previa que integre, organice y articule los aspectos que se trabajan desde las diferentes disciplinas.

Al interior de este análisis, un cuarto elemento estructural derivado de la revisión de los 59 documentos oficiales, se refiere a la incorporación del problema ambiental en la estructura del Plan de Estudio, registrando que ninguna de las Instituciones precisa en ello, por el contrario lucen en común un distintivo orientado en mostrar calendarios ambientales asociados a fechas específicas y a la participación de las áreas que ponen en quiebre la esencia de la educación ambiental en el plan de estudios, centran la atención en asuntos superficiales y en 
particular, insisten en el abordaje asignaturista y en solitario de la problemática ambiental (tendencia a residuos sólidos) lejos de una dinámica integradora y formativa.

Concomitante con el aspecto anterior, un quinto y último elemento estructural es el relacionado con el papel que juega el PRAE en los Planes de Mejoramiento Institucional, destacando que solo el $6,77 \%$ (4), lo precisa y con ello, pone en relieve la concordancia en atender de modo integrado y articulador las problemáticas ambientales como una política institucional. Se trata por tanto de insistir en estos acuerdos que sostienen los horizontes de la escuela proyectada a los ámbitos sociales, permitiéndole un acercamiento a la realidad de la comunidad para intervenirla, comprenderla y plantear solución los problemas ambientales; y que legitima a los PRAEs como herramienta que posibilita en los alumnos el pensamiento crítico, reflexivo y el desarrollo de actitudes y acciones coherentes con su realizada contextual.

Algunos aspectos para destacar en las entrevistas con los grupos focales, se relaciona con el hecho de que algunos PRAEs han contado con el apoyo de Universidades, de la Policía Ambiental y de la Corporación Autónoma de los Valles del Sinú y San Jorge, sin embargo, en la mayoría de los documentos analizados se no se visibilizan procesos de gestión interinstitucional en la búsqueda de recursos financieros o de apoyo logístico que brinden la oportunidad de darle continuidad a los proyectos, que además, se proyecten a la ciudadanía y lograr su incorporación como PROCEDA, o en los planes de desarrollo e incluso en el plan de ordenamiento territorial.

\section{CONSIDERACIONES FINALES}

Los resultados muestran que los PRAEs se han diseñado y ejecutado con poca participación y reflexión política, ética, económica y social dejando de lado su carácter transversal e interdisciplinario. Este primer acercamiento da cuenta de que la educación ambiental desarrollada en estos proyectos es una tarea inconclusa, lo que provoca serios llamados a continuar en el estudio del estado actual de los PRAEs, trascendiendo a una nueva etapa de valoraciones donde lo documental se cruce con las actuaciones y con ello, nuevos retos frente al lugar del PRAE en la escuela, atendiendo a que desde este campo de formación se requiere el desarrollo de actitudes y comportamientos que lleven a una relación armónica con la naturaleza, así como el impulso de las capacidades necesarias para poder actuar individual $y$ colectivamente.

Otro aspecto, asociado a la tarea inclusa en los PRAEs, es la transversalización como un modelo curricular que lleve a la integración de las distintas áreas de enseñanza que se dinamizan en el proyecto educativo institucional, que propenda por un saber práctico con aprendizajes significativos que contribuya a la formación ambiental y al fortalecimiento de valores de solidaridad, tolerancia, autonomía y austeridad a través de la problematización de los paradigmas dominantes y la incorporación del saber ambiental en el currículo hacia a una formación integral del estudiante llevándolo a que se comprometa con el mejoramiento de la calidad de vida. Otro aspecto en relación con la dinamización y que fue ratificado por los docentes entrevistados es el hecho de que en su implementación efectiva solo participan pequeños grupos, es decir que no se hace extensivo a todo el estudiantado, lo que deja de lado su compromiso con la formación integral.

Un aspecto que le hace falta a los PRAEs del municipio de Montería es la proyección socialcomunitaria prevista en el marco normativo de la Educación Ambiental, estos proyectos no han logrado irradiarse a su contexto local y regional; lo que muestra debilidades en las etapas de formulación, desarrollo e impacto quedando 
inconclusa la formación ambiental. No se hacen evidentes cambios en prácticas y acciones individuales y colectivas respecto al pensar, sentir y actuar armónicamente con el ambiente en relación con procesos formativos ambientales que impacten en las expectativas de vida y el bienestar comunitario, lo que se convierte en insumo importante que lleve a realizar cambios $y$ ajustes a los PRAEs en el desarrollo de una efectiva Educación Ambiental.

\section{REFERENCIAS BIBLIOGRÁFICAS}

Andrade, L., y Andrade, M. (2017). Los Proyectos Ambientales Escolares: una alternativa para la Educación Ambiental. Revista Biocenosis, 31 (2), 18, 28

Burgos Ayala, A. (2017) Estado de los proyectos ambientales escolares en Boyacá. Revista Luna Azul, 44, 39-58. DOI: 10.17151/luaz.2017.44.4

Burgos, A. (2013). La educación ambiental: impacto y conocimiento de sus fines y formas. Cultura Científica, 11, 20-31.

Bustamante, N., Cruz, M., y Vergara Rivera. (2017). Proyectos ambientales escolares y la cultura ambiental en la comunidad estudiantil de las instituciones educativas de Sincelejo, Colombia. Revista Logos Ciencia \& Tecnología, 9(1), 215-229.

Decreto 1743 de 1994. Por el cual se instituye el Proyecto de Educación Ambiental para todos los niveles de educación formal, se fijan criterios para la promoción de la educación ambiental no formal e informal y se establecen los mecanismos de coordinación entre el Ministerio de Educación nacional y el Ministerio del Medio Ambiente. 5 de agosto de 1994 D.O No 41.476 .
Eschenhagen, M. (2003). Estado del arte de la Educación Ambiental y problemas a los que se está enfrentando. En: Memorias II Encuentro Metropolitano de Educación Ambiental. México.

Flórez Restrepo, G. (2012). La educación ambiental: una apuesta hacia la integración escuela-comunidad. Praxis \& Saber, 3(5),79-101.

González Pachón, R. (2014). Análisis de la incidencia de los proyectos educativos ambientales en la Institución Educativa San Víctor. Período 2008-2013. Desde la perspectiva socioambiental. [Trabajo de Maestría, Universidad de Manizales]

Gutiérrez Sabogal, L. H. (2017). La educación ambiental: una estrategia didáctica para favorecer el conocimiento escolar deseable en educación básica secundaria en la Institución Educativa Departamental Ignacio Pescador de Choachí Cundinamarca. [Tesis Doctoral. Universidad La Salle- Colombia].

Leff, E. (2019). Ecología política. De la deconstrucción del capital a la territorialización de la vida. Siglo XXI Editores.

Leff, E. (2008). Discursos Sustentables. Siglo XXI Editores

Mora-Ortiz, J. (2015) Los Proyectos Ambientales Escolares. Herramientas de gestión ambiental. Revista Bitácora Urbano Territorial, 25(2), 67-74

Ministerio de Educación Nacional de Colombia -MEN-y Ministerio del Medio Ambiente de Colombia -MMA-. (2002). Política Nacional de Educación Ambiental.

Ministerio de Educación Nacional (2011). Subdirección de Articulación Educativa e 
Intersectorial - Programa de Educación Ambiental. Módulo PRAEs.

Monzón, L. (2011). La identidad docente desde una perspectiva hermenéutica. Revista de Educación y Desarrollo, 18, 27-34

Pasek De Pinto, E. (2004). Hacia una conciencia ambiental. Revista Educare, 8(24), 3440

Perez-Vásquez, N. (2020). Educación Ambiental de docentes en formación a partir de una propuesta curricular alternativa soportada en la interdisciplinariedad y la responsabilidad ética, política y social. [Tesis doctoral, Universidad Surcolombiana]

Torres Carrasco, M. (2002). Estudios ambientales escolares. MEN. Santa Fe de Bogotá.

Villadiego Lorduy, J., Cardona, C., Ortiz, R., Coneo, M. y Ramos, A. (2011). La EducaciónAmbiental en el departamento de Córdoba: El caso de los proyectos ambientales escolares PRAE. Revista de Didáctica Ambiental,10, 7-15. 